we next examined the effect of SP600125 on grafted embryonic DA neurons in vivo, in an animal model of PD. We unilaterally injected the neurotoxin 6-OHDA into the right SNpc of rats to produce a complete ablation of cell bodies as well as fibers and terminals innervating the striatum. Based on previous findings, ${ }^{20}$ all animals showing greater than 5 rotations per minute were assumed to have lesions greater than $90 \%$ and were selected for transplantation. At 2 weeks post-lesioning, $400000 \mathrm{VM}$ cells were treated with vehicle or SP600125 and grafted into the striatum of lesioned rats. ${ }^{20}$ Animals received daily infusions of vehicle or SP600125 $(75 \mu \mathrm{M})$ via a cannula in the striatum for 4 days and were killed 7 days post-transplantation. Striatal examination revealed that $100 \%$ of the VM grafts survived in animals infused with either vehicle or SP600125. However, total counts of $\mathrm{TH}+$ cells in the grafts of animals infused with SP600125 showed significantly more (231\%) DA cells than in the grafts of animals infused with vehicle (mean \pm S.E.M, $12285 \pm 3436$ and $28406 \pm 5506$, respectively, Figures $1 \mathrm{e}(\mathrm{i})$ and (f)). Thus, treatment of cells with SP600125 upon transplantation into 6-OHDA lesioned brains results in a two-fold increase in the survival of grafted DA neurons within the host striatum. Moreover, we found that within 7 days, these cells had adopted a typical bipolar DA phenotype with extensive processes, verifying that the cells incorporated within the host and did not form tumors (Figure 1e(ii)). These results indicate that SP600125 selectively prevents cell death, and does not induce excessive proliferation in vivo. From a clinical viewpoint, our results imply that the amount of DA precursors needed for transplantation in PD could be at least halved by using the SP600125 compound. Taken together, our study indicates that inhibition of apoptotic JNK signaling may contribute to overcome one of the major limitations of current cell replacement strategies in the treatment of PD.
Acknowledgements. We thank Dr. Tibor Harkany for fruitful discussions, Dr. Björn Schilström for technical advice and Claudia Tellos-Lagos for technical assistance. Financial support was obtained from the Swedish Foundation for Strategic Research, EuroStemCell, Swedish Royal Academy of Sciences, Knut and Alice Wallenberg Foundation, and the Karolinska Institute. CP was supported by the Human Frontiers Scientific Program Longterm Fellowship, and GCB was supported by the Praxis XXI programme of the Portuguese Fundação para a Ciência e Tecnologia/European Social Fund, the Karolinska Institute, and Calouste Gulbenkian Foundation.

\section{N Rawal ${ }^{1}, C$ Parish $^{1,2}$, G Castelo-Branco ${ }^{1,2}$ and E Arenas $^{* 1}$}

${ }^{1}$ Laboratory of Molecular Neurobiology, Department of Medical Biochemistry and Biophysics, Karolinska Institute, Scheeles v. 1, A1:2, 17177 Stockholm Sweden

2 These authors contributed equally to this work.

* Corresponding author: E Arenas, Laboratory of Molecular Neurobiology, Department of Medical Biochemistry and Biophysics, Karolinska Institute, Scheeles v. 1, A1:2, 17177 Stockholm, Sweden. Tel: + 46852487663; Fax: + 468341960; E-mail: Ernest.Arenas@ki.se

1. de Rijk MC et al. Neurology 2000; 54 (Suppl 5): S21-S23.

2. Lindvall $O$ et al. Ann Neurol 1994; 35: 172-180.

3. Piccini $P$ et al. Nat Neurosci 1999; 2: 1137-1140.

4. Wenning GK et al. Ann Neurol 1997; 42: 95-107.

5. Olanow CW et al. Trends Neurosci 1996; 19: 102-109.

6. Lindvall O, Bjorklund A. NeuroRx 2004; 1: 382-393.

7. Madrazo I et al. N Engl J Med 1988; 318: 51

8. Quinn NP. Prog Brain Res 1990; 82: 619-625

9. Brundin $P$ et al. Cell Transplant 2000; 9: 179-195

10. Zeng X et al. Stem Cells 2004; 22: 925-940.

11. Barker RA et al. Exp Neurol 1996; 141: 79-93.

12. Zawada WM et al. Brain Res 1998; 786: 96-103.

13. Schierle GS et al. Nat Med 1999; 5: 97-100.

14. Silva RM et al. Mov Disord 2005; 20: 653-664.

15. Holtz WA, O'Malley KL. J Biol Chem 2003; 278: 19367-19377.

16. Vaudano E, Rosenblad C, Bjorklund A. Eur J Neurosci 2001; 13: 1-14.

17. Xia XG et al. Proc Natl Acad Sci USA 2001; 98: 10433-10438.

18. Wang W et al. Neurosci Res 2004; 48: 195-202.

19. Bennett BL et al. Proc Natl Acad Sci USA 2001; 98: 13681-13686.

20. Parish CL et al. Stem Cells 2005; 23: 471-476.

\title{
Mitochondrial membrane depolarization in Drosophila apoptosis
}

\author{
Cell Death and Differentiation (2007) 14, 383-385. doi:10.1038/sj.cdd.4402036; published online 15 September 2006
}

\section{Dear Editor}

Apoptosis regulation in Drosophila has many parallels to vertebrate systems. However, one major difference has been the apparent lack of mitochondrial significance in Drosophila cell death. Several reports have suggested that mitochondrial outer membrane permeabilization does not occur in Drosophila apoptosis and that there is no significant mitochondrial contribution to apoptosis in the fly. ${ }^{1-6}$ However, these findings are based largely, if not exclusively, on studies in cultured S2 cells, which are phagocytic cells derived from embryonic macrophages/hemocytes. ${ }^{7,8}$ The specialized nature of S2 cells prompted us to consider whether they are generally representative of Drosophila cells in their response to apoptotic stimuli. We addressed this question by examining the response of embryonic macrophages to apoptotic signals in situ in the embryo.

In keeping with previous accounts, we find that apoptotic S2 cells maintain mitochondrial membrane integrity as measured by CMXRos staining. ${ }^{4}$ CMXRos is a mitochondrial-specific label dependent on membrane polarization for uptake. Untreated cells show punctate cytoplasmic staining consistent 

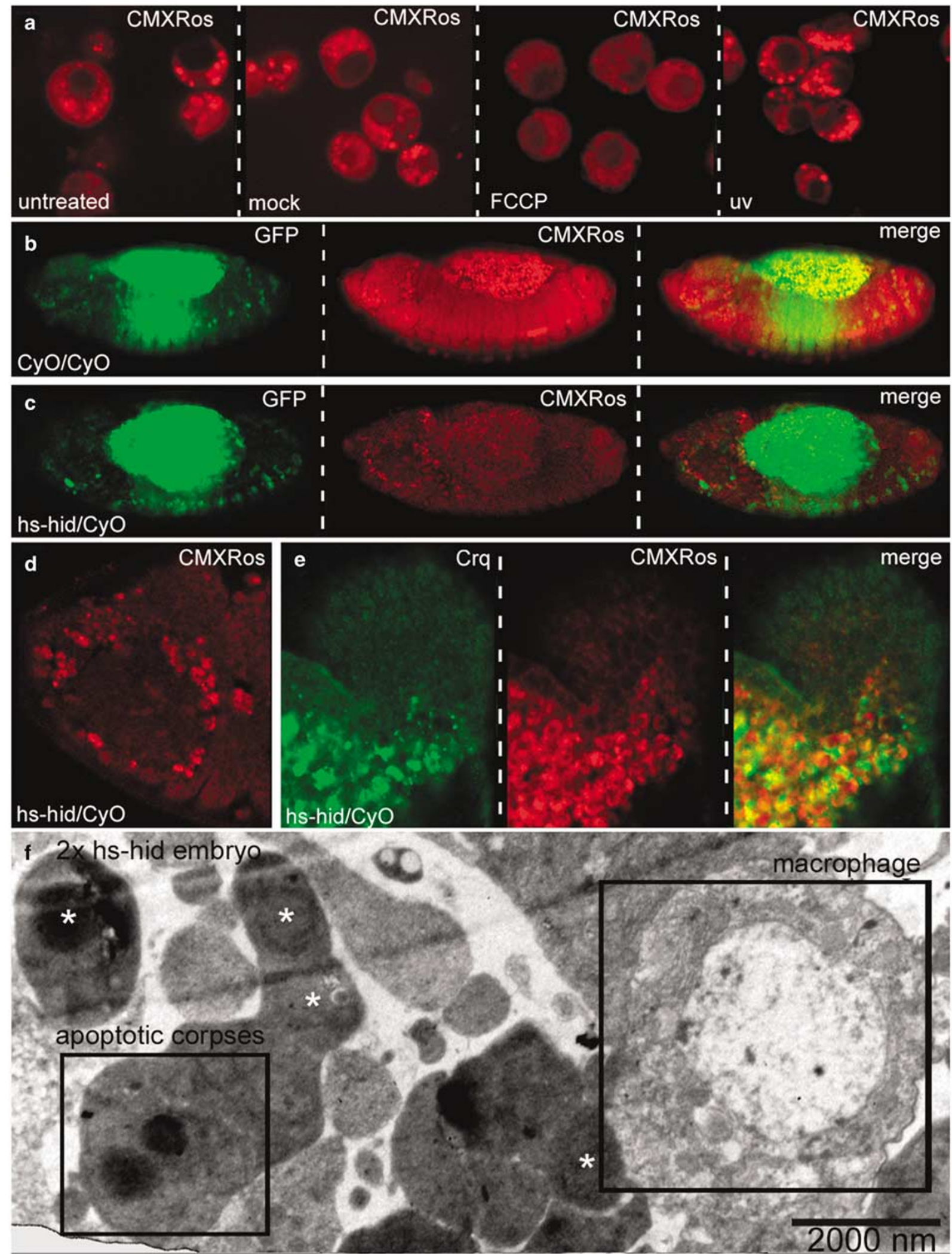

with mitochondrial labeling (Figure 1a). The specificity of labeling is demonstrated by treatment with carbonyl cyanide $p$-trifluoromethoxy-phenylhydrazone (FCCP), a protonophore that depolarizes mitochondria by abolishing the proton gradient across the inner mitochondrial membrane ${ }^{9,10} \mathrm{~S} 2$ cells continue to stain with CMXRos qat high levels even after exposure to UV 
Figure 1 Embryonic macrophages selectively maintain mitochondrial membrane polarization following induction of apoptosis. (a) S2 cells stained with CMXRos: untreated, mock treated, FCCP treated, UV-irradiated. (b, c) Stage 13 embryos stained with CMXRos and imaged live through the vitelline membrane. (b) CyO/CyO, (c) hs-hid/CyO. Embryos in ( $\mathbf{b}$ and $\mathbf{c}$ ) were subjected to 60 min of heat shock before staining. Embryos in ( $\mathbf{b}$ and $\mathbf{c}$ ) are siblings and were prepared and imaged together. Genotypes were determined by the intensity of green fluorescent protein (GFP) from a kruppel-GFP CyO balancer. (d) Head region of embryo shown in (c) showing CMXRos-positive macrophages in a field of CMXRos-negative cells. (e) hs-hid/CyO embryo subjected to 60 min of heat shock and labeled with CMXRos and anti-Crq to confirm the identity of CMXRos-positive cells. Image is of the extreme anterior portion of the embryonic head. (f) TEM of $90 \mathrm{~nm}$ thin section through the head region of a stage $13 \mathrm{hs}$-hid embryo subjected to $60 \mathrm{~min}$ of heat shock before fixation. Low-magnification image of region showing a healthy macrophage (large cell, boxed) surrounded by apoptotic corpses (small cell boxed; asterisks). Higher magnification images of boxed cells are shown in Supplementary Figure 1

irradiation and a recovery period of $6 \mathrm{~h}$ (Figure 1a). However, this is not the case with Drosophila cells in general. Embryos subjected to ectopic hid expression (Figure 1c-e) or UV irradiation (not shown) rapidly lose the capacity to take up CMXRos in nearly all cells. Within $1 \mathrm{~h}$ of the initiation of either signal, nearly all cells of the embryo fail to stain with CMXRos. The notable exception is macrophages, which continue to stain at high levels whereas other cell types do not (Figure 1c-d). These cells are identifiable as macrophages by their stereotypic distribution and enormous size, and by doubly labeling for Croquemort (Figure 1e), a macrophage-specific cell surface marker. ${ }^{11,12}$ Identical results were obtained using the mitochondrial marker TMRE (not shown).

These findings suggest that macrophages have the capacity to remain healthy in the presence of apoptotic signals that readily affect other cell types. We addressed this possibility by examining the ultrastructure of macrophages in apoptotic embryos by transmission electron microscopy. We found that after $1 \mathrm{~h}$ of heat shock-induced hid expression, macrophages in the embryonic head showed no overt signs of apoptosis, while surrounded by apoptotic corpses derived from other cell types (Figure 1f; Supplementary Figure 1). Macrophage chromatin remained uncondensed with the nuclear envelope intact, and membranebound organelles including mitochondria, Golgi and endoplasmic reticulum were clearly visible in the cytoplasm. By contrast, surrounding corpses showed condensed chromatin, degeneration of the nuclear envelope and loss of subcellular organelles (Figure 1f; Supplementary Figure 1). These results are consistent with observations by us and others that following exposure to UV irradiation, Croquemort-positive cells in the embryo become quite large owing to multiple engulfment events ( $R$ Hays, unpublished finding). ${ }^{11}$ One would expect macrophages to undergo apoptosis like any other cell type, having been exposed to the same dose of radiation. However, they survive to engulf corpses for a period of at least several hours.

These findings suggest that macrophages are uniquely resistant to apoptosis among embryonic cell types, and that S2 cells, an embryonic macrophage line, is not a suitable general model for Drosophila cell death. They further suggest that apoptosis regulation in Drosophila has more in common with vertebrate systems than previously appreciated, and re-shape a large segment of current Drosophila apoptosis models.

Acknowledgements. We are grateful to $\mathrm{A}$ Ezekowitz and $\mathrm{R}$ Ward for reagents. This work was supported by NIH 2 P20 RR016475-04.

\section{JC Means ${ }^{1}$ and $R$ Hays $^{1}$}

Division of Biological Sciences, University of Kansas, 1200 Sunnyside Avenue, Lawrence, KS, USA

Corresponding author: R Hays, Division of Biological Sciences, University of Kansas, 1200 Sunnyside Avenue, Lawrence, KS 66045, USA.

Tel. + 1-785-864-5744; Fax; + 1-785-864-5294;

E-mail: rebahays@ku.edu

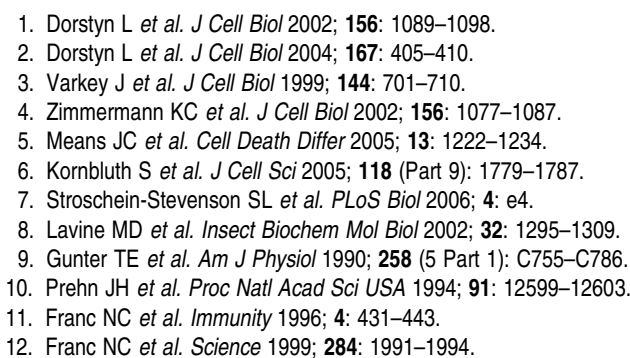

Supplementary Information accompanies the paper on Cell Death and Differentiation website (http://www.nature.com/cdd)

\title{
Cavitation of embryoid bodies requires optimal oxidative phosphorylation and AIF
}

\author{
Cell Death and Differentiation (2007) 14, 385-387. doi:10.1038/sj.cdd.4402041; published online 29 September 2006
}

\section{Dear Editor,}

Apoptosis inducing factor (AIF), which is encoded by the $p c d 8$ gene on the $\mathrm{X}$ chromosome, is a protein that is normally present in mitochondria. ${ }^{1,2}$ The AIF flavoprotein can participate in the scavenging of reactive oxygen species, ${ }^{3}$ and 